\title{
Global well-posedness of a conservative relaxed cross diffusion system.
}

\author{
Thomas Lepoutre $\quad$ Michel Pierre Guillaume Rolland
}

March 29, 2022

\begin{abstract}
AMS subject classification: Primary 35K51, 35K57, 35K59; Secondary 92D25.
Keywords: reaction-diffusion, cross-diffusion, quasilinear systems, global existence, population dynamics.

Abstract

We prove global existence in time of solutions to relaxed conservative cross diffusion systems governed by nonlinear operators of the form $u_{i} \rightarrow \partial_{t} u_{i}-\Delta\left(a_{i}(\tilde{u}) u_{i}\right)$ where the $u_{i}, i=1, \ldots, I$ represent $I$ density-functions, $\tilde{u}$ is a spatially regularized form of $\left(u_{1}, \ldots, u_{I}\right)$ and the nonlinearities $a_{i}$ are merely assumed to be continuous and bounded from below. Existence of global weak solutions is obtained in any space dimension. Solutions are proved to be regular and unique when the $a_{i}$ are locally Lipschitz continuous.
\end{abstract}

\section{Introduction}

Introduced by Shigesada et al. [28], cross diffusion models try to represent the effect of the interaction between species through motion, and not only as usual through reaction. These models have been studied by Levin [18], Levin and Segel [17], Okubo [26], Mimura and Murray [23], Mimura and Kawasaki [22], Mimura and Yamaguti [24], Andreianov et al. [1], Bendahmane and Langlais [2] and many other authors: a survey by A. Jüngel may be found in [12] for applications to population dynamics. In those references, a general system is the following:

$$
\left\{\begin{array}{l}
\partial_{t} u_{1}-\Delta\left[u_{1}\left(d_{1}+d_{11} u_{1}^{p}+d_{12} u_{2}^{p}\right)\right]=r_{1}\left(u_{1}, u_{2}\right), \\
\partial_{t} u_{2}-\Delta\left[u_{2}\left(d_{2}+d_{21} u_{1}^{p}+d_{22} u_{2}^{p}\right)\right]=r_{2}\left(u_{1}, u_{2}\right), \\
\partial_{n}\left[u_{1}\left(d_{1}+d_{11} u_{1}^{p}+d_{12} u_{2}^{p}\right)\right]=\partial_{n}\left[u_{2}\left(d_{2}+d_{21} u_{1}^{p}+d_{22} u_{2}^{p}\right)\right]=0 .
\end{array}\right.
$$

For the system (1) with $p=1$ and Lotka-Volterra-type reaction, there exists a wide literature, studying specific cases of the system where an additional structure keeps it parabolic or with cross diffusion pressure only on one of the species (see e.g. Wang [29] and the many references therein, especially in the introduction). To our knowledge, the most general result on global weak solutions might be found in Chen and Jüngel [7] where the entropy structure of the model is used. For existence of classical solutions the reader might consult [29, 19] by Wang and Li-Zhao for instance. In population dynamics, one of the most interesting features of cross diffusion is its effect on steady states: cross diffusion pressure might help the appearance of nonconstant steady states when the reaction structure does not drive to segregation (see Iida-Mimura-Ninomyia [11] for instance). However, in these cases, 
the pattern formation relies on the reaction term (for instance, the convergence to homogeneous steady states in absence of reaction is proved in [7]).

In [3], [15], the first author and collaborators introduced a relaxation of conservative cross diffusion systems, replacing

$$
\left\{\begin{array}{l}
\partial_{t} u_{i}-\Delta\left[a_{i}(u) u_{i}\right]=0, \text { on }(0,+\infty) \times \Omega, \Omega \subset \mathbb{R}^{N}, \text { bounded } \\
u=\left(u_{1}, \ldots, u_{I}\right) \\
\partial_{n}\left[a_{i}(u) u_{i}\right]=0 \text { on }(0,+\infty) \times \partial \Omega, \quad u(0, \cdot)=u^{0} \text { given, }
\end{array}\right.
$$

where $a_{i}:[0, \infty)^{I} \rightarrow[0, \infty)$, by the following relaxed model:

$$
\left\{\begin{array}{l}
\partial_{t} u_{i}-\Delta\left[a_{i}(\tilde{u}) u_{i}\right]=0, \text { on }(0,+\infty) \times \Omega, \\
u=\left(u_{1}, \ldots u_{I}\right), \\
\tilde{u}_{i}-\delta_{i} \Delta \tilde{u}_{i}=u_{i}, \text { on }(0,+\infty) \times \Omega, \quad \delta_{i}>0, \\
\partial_{n} u_{i}=\partial_{n} \tilde{u}_{i}=0 \text { on }(0,+\infty) \times \Omega, \quad u(0, \cdot)=u^{0} \text { given. }
\end{array}\right.
$$

This model was introduced in order to investigate the effect of non classical cross diffusion pressure on the segregative behavior (and $a_{i}(\cdot)$ is often truly nonlinear). One of the purposes was to drive spatial segregation only through motion. Its effects on the stability of the homogeneous equilibria is investigated in $[15,3,16]$. This relaxed version is also relevant in some applications: it takes into account that the intensity of the underlying Brownian motion depends on the density of the population measured with a space scale $\delta_{i}$ and not exactly at the exact location $x$. It takes therefore into account the fact that a species can react to the presence of another species in a neighborhood.

Models with nonlocal diffusion coefficients can be seen also in [4] (where the self-diffusion coefficients depend on the total population). Nonlocal reaction terms can also be considered ([5, 8, 25] for instance), but the goal of our model is more to create patterns only through motion.

A first well-posedness result for the relaxed model was derived in [15], [3] in dimension $N=1,2$ and with some restrictions on the structure of the nonlinearities $a_{i}$ (basically, the $a_{i}$ are $C^{2}$ and have at most a polynomial growth in $u$ ). In this paper, we prove existence of solutions for this system in any dimension and for general nonlinearities $a_{i}$, which are only assumed to be continuous and bounded from below. Weak solutions are obtained in general and they are proved to be strong and unique as soon as the $a_{i}$ are locally Lipschitz continuous. Some $L^{2}$-estimates are exploited in the spirit of [27] to prove existence of weak solutions. A main point is that $\tilde{u}$ is uniformly bounded in any dimension for these weak solutions. Next, one has to deal with parabolic operators of the form $u_{i} \rightarrow \partial_{t} u_{i}-\Delta\left(a_{i}(\tilde{u}) u_{i}\right)$ : they are not of divergence form, but they are uniformly parabolic since $a_{i}(\tilde{u})$ is then bounded from above and from below. Using the $C^{\alpha}$-theory for the duals of these operators, namely $U_{i} \rightarrow \partial_{t} U_{i}-a_{i}(\tilde{u}) \Delta U_{i}$, in the spirit of Krylov-Safonov [13], [10] (see also the book by Lieberman [20]), we prove that $\tilde{u}$ is even Hölder-continuous. This provides continuous coefficients $a_{i}(\tilde{u})$ for the above operators, and then, $L^{p}$-estimates classically follow for the solution. When the $a_{i}$ are locally Lipschitz continuous, even $\partial_{t} u_{i}, \Delta\left(a_{i}(\tilde{u}) u_{i}\right)$ are proved to be in $L^{p}$ so that the solution is strong: moreover, weak solutions are then proved to be unique.

Let us fix the notations and state the main result. We assume that $\Omega \subset \mathbb{R}^{N}$ is a bounded subset with a $C^{2}$-boundary. The exterior normal derivative operator on $\partial \Omega$ is denoted by $\partial_{n}$. For all $T>0$, we denote $Q_{T}=(0, T) \times \Omega, \Sigma_{T}=(0, T) \times \partial \Omega$. For $\alpha \in(0,1]$, we denote

$$
C^{\alpha}\left(Q_{T}\right)=\left\{v \in L^{\infty}\left(Q_{T}\right) ;\|v\|_{T}^{(\alpha)}<+\infty\right\},
$$




$$
\|v\|_{T}^{(\alpha)}=\|v\|_{L^{\infty}\left(Q_{T}\right)}+\sup \left\{\frac{|v(t, x)-v(s, y)|}{\left[|t-s|+|x-y|^{2}\right]^{\frac{\alpha}{2}}},(t, x),(s, y) \in Q_{T}\right\} .
$$

We will at least assume that

$$
\forall i=1, \ldots, I, \quad a_{i}:[0, \infty)^{I} \rightarrow[0, \infty) \text { is continuous and }: \inf _{r \in[0, \infty)^{I}} a_{i}(r) \geq \underline{d}>0
$$

And we are given $\delta_{i} \in(0, \infty), \forall i=1, \ldots I$.

Theorem 1.1 Assume (3) and $u^{0}=\left(u_{1}^{0}, \ldots, u_{I}^{0}\right) \in L^{\infty}(\Omega,[0, \infty))^{I}$. Then, there exists a nonnegative solution $u=\left(u_{1}, \ldots, u_{I}\right)$ to the following problem:

$$
\left\{\begin{array}{l}
\forall T \in(0, \infty), \forall i=1, \ldots, I, \forall p \in[1, \infty) \\
u_{i} \in L^{p}\left(Q_{T}\right) ; \tilde{u}_{i} \in C^{\alpha}\left(Q_{T}\right) \cap L^{p}\left(0, T ; W^{2, p}(\Omega)\right) \text { for some } \alpha \in(0,1] \\
\int_{0}^{t} a_{i}(\tilde{u}) u_{i} \in L^{p}\left(0, T ; W^{2, p}(\Omega)\right) \\
u_{i}(t)-\Delta\left[\int_{0}^{t} a_{i}(\tilde{u}) u_{i}\right]=u_{i}^{0} \text { in } Q_{T} \\
\tilde{u}_{i}-\delta_{i} \Delta \tilde{u}_{i}=u_{i} \text { in } Q_{T} \\
\partial_{n}\left(\int_{0}^{t} a_{i}(\tilde{u}) u_{i}\right)=0=\partial_{n} \tilde{u}_{i} \text { on } \Sigma_{T} .
\end{array}\right.
$$

If moreover

$$
\forall i=1, \ldots, I, a_{i}:[0, \infty)^{I} \rightarrow[0, \infty) \text { is locally Lipschitz continuous }
$$

then, $\forall i=1, \ldots, I, \forall T>0, \forall p \in[1, \infty)$,

$$
u_{i} \in L^{\infty}\left(Q_{T}\right), \forall \tau \in(0, T), \partial_{t} u_{i}, \Delta\left(a_{i}(\tilde{u}) u_{i}\right) \in L^{p}((\tau, T) \times \Omega)
$$

and $\partial_{t} u_{i}-\Delta\left(a_{i}(\tilde{u}) u_{i}\right)=0, \partial_{n}\left(a_{i}(\tilde{u}) u_{i}\right)=0$ in a pointwise sense. Finally, under assumption (5), solutions of (4) are unique.

The paper is organized as follows.

Section 2 first assumes that the nonlinearities $a_{i}$ are also bounded from above. We prove existence of a weak solution to the system (4) by a standard Leray-Schauder fixed-point argument. The underlying space is an adequate subspace of $L^{2}\left(Q_{T}\right)$ and the required compactness follows essentially from Lemma 2.3 .

Section 3 is devoted to the proof of the $L^{\infty}$-estimate on $\tilde{u}$. Then, the assumption of the bound from above on the $a_{i}$ may be dropped.

Section 4 exploits this $L^{\infty}$-estimate to prove that the weak solution is actually rather regular, and existence as stated in Theorem 1.1 follows. The $C^{\alpha}$-theory for nondivergential parabolic operators is used there. An alternative more elementary proof of the regularity is also given when monotonicity properties hold for the $a_{i}$ together with locally Lipschitz continuity.

The uniqueness stated in Theorem 1.1 is proved in Section 5. It is based on solving an original dual problem, interesting for itself.

A short Section 6 indicates without proof a complementary approach which provides a constructive and alternative way of proving existence of a solution and which may be used to compute it numerically. 


\section{Global existence when $a_{i}$ is bounded}

In this section, we first prove existence of weak-solutions of (4) on a given interval $[0, T]$ when, besides (3), the nonlinearities $a_{i}$ also satisfy

$$
\exists \bar{d}>0, \quad \forall i=1, \ldots, I, \quad \sup _{r \in[0, \infty)^{I}} a_{i}(r) \leq \bar{d} .
$$

Proposition 2.1 Let $T>0$. Assume (3), (6) and $\forall i=1, \ldots, I, u_{i}^{0} \in L^{2}(\Omega ;[0, \infty))$. Then, there exists a nonnegative solution $u=\left(u_{1}, \ldots, u_{I}\right)$ to the system

$$
\left\{\begin{array}{l}
\forall i=1, \ldots, I, \\
u_{i} \in L^{2}\left(Q_{T}\right), \int_{0}^{t} a_{i}(\tilde{u}) u_{i} \in L^{2}\left(0, T ; H^{2}(\Omega)\right) \\
\tilde{u}_{i} \in L^{2}\left(0, T ; H^{2}(\Omega)\right), \tilde{u}_{i}-\delta_{i} \Delta \tilde{u}_{i}=u_{i} \text { on } Q_{T}, \tilde{u}_{i} \geq 0 \\
u_{i}-\Delta\left(\int_{0}^{t} a_{i}(\tilde{u}) u_{i}\right)=u_{i}^{0} \text { on } Q_{T} \\
\partial_{n} \tilde{u}_{i}=0=\partial_{n}\left(\int_{0}^{t} a_{i}(\tilde{u}) u_{i}\right) \text { on } \Sigma_{T} .
\end{array}\right.
$$

To prove Proposition 2.1, we will use the classical Leray-Schauder's approach, namely (see e.g. [9], Theorem 11.3)

Lemma 2.2 (Leray-Schauder) Let $\left(X,\|\cdot\|_{X}\right)$ be a Banach space and $\mathcal{T}: X \rightarrow X$ a continuous compact mapping. Suppose that

$$
\exists M>0, \forall \sigma \in[0,1],[u \in X, u=\sigma \mathcal{T} u] \Rightarrow\left[\|u\|_{X} \leq M\right] .
$$

Then, there exists $u \in X$ such that $u=\mathcal{T} u$.

To define the mapping $\mathcal{T}$, we will use the following lemma.

Lemma 2.3 Let $T>0, w_{0} \in L^{2}(\Omega ;[0,+\infty)), A \in L^{\infty}\left(Q_{T}\right), \underline{a}, \bar{a} \in(0, \infty)$ such that $0<\underline{a} \leq A \leq$ $\bar{a}<+\infty$. Then there exists a unique nonnegative solution $w=w\left(A, w_{0}\right)$ to

$$
\left\{\begin{array}{l}
w \in L^{2}\left(Q_{T}\right), \quad \int_{0}^{t} A w \in L^{2}\left(0, T ; H^{2}(\Omega)\right) \\
w-\Delta\left(\int_{0}^{t} A w\right)=w_{0} \text { on } Q_{T}, \partial_{n}\left(\int_{0}^{t} A w\right)=0 \text { on } \Sigma_{T}
\end{array}\right.
$$

Moreover, if

$$
A^{n} \in L^{\infty}\left(Q_{T}\right), 0<\underline{a} \leq A^{n} \leq \bar{a}<\infty, A^{n} \rightarrow \text { A a.e., } w_{0}^{n} \rightarrow w_{0} \text { in } L^{2}(\Omega),
$$

then $w\left(A^{n}, w_{0}^{n}\right)$ converges strongly in $L^{2}\left(Q_{T}\right)$ to $w\left(A, w_{0}\right)$.

Proof of Lemma 2.3: Using convolution, we approximate $A$ by a sequence of smooth functions $\left(A^{n}\right)_{n \in \mathbb{N}} \in C^{\infty}\left(\overline{Q_{T}}\right)$ such that $\underline{a} \leq A^{n} \leq \bar{a}$ and $A^{n} \rightarrow A$ a.e.. Let also $w_{0}^{n}$ be a regular approximation of $w_{0}$. There exists a classical regular nonnegative solution $w^{n}$ of (see e.g. [14], Theorem V.7.4, applied to the unknown $A^{n} w^{n}$ )

$$
\partial_{t} w^{n}-\Delta\left(A^{n} w^{n}\right)=0 \text { on } Q_{T}, \partial_{n}\left(A^{n} w^{n}\right)=0 \text { on } \Sigma_{T}, w^{n}(0, \cdot)=w_{0}^{n} .
$$


Integrating (9) in time gives

$$
w^{n}(t)-\Delta\left(\int_{0}^{t} A^{n} w^{n}\right)=w_{0}^{n} \text { on } Q_{T}, \partial_{n}\left(\int_{0}^{t} A^{n} w^{n}\right)=0 \text { on } \Sigma_{T} .
$$

We multiply by $A^{n} w^{n}$ and use the following identity, valid for $z^{n}=A^{n} w^{n}$ :

$$
-\int_{\Omega} z^{n} \Delta \int_{0}^{t} z^{n}=\int_{\Omega} \nabla z^{n} \nabla \int_{0}^{t} z^{n}=\int_{\Omega} \frac{1}{2} \partial_{t}\left|\nabla \int_{0}^{t} z^{n}\right|^{2} .
$$

We obtain the following estimate after integration in time

$$
\int_{Q_{T}} A^{n}\left(w^{n}\right)^{2}+\int_{\Omega} \frac{1}{2}\left|\nabla \int_{0}^{T} A^{n} w^{n}\right|^{2}=\int_{Q_{T}} w_{0}^{n} A^{n} w^{n} .
$$

In particular

$$
\underline{a} \int_{Q_{T}}\left(w^{n}\right)^{2} \leq \bar{a} \sqrt{T}\left(\int_{\Omega}\left(w_{0}^{n}\right)^{2}\right)^{1 / 2}\left(\int_{Q_{T}}\left(w^{n}\right)^{2}\right)^{1 / 2} \Rightarrow \underline{a}\left\|w^{n}\right\|_{L^{2}\left(Q_{T}\right)} \leq \bar{a} \sqrt{T}\left\|w_{0}^{n}\right\|_{L^{2}(\Omega)} .
$$

Now, up to a subsequence, $w^{n}$ converges weakly in $L^{2}\left(Q_{T}\right)$ to some $w$. By the pointwise and uniformly bounded convergence of $A^{n}$ to $A$, for all $\psi \in L^{2}\left(Q_{T}\right), \psi A^{n}$ converges strongly in $L^{2}\left(Q_{T}\right)$ to $\psi A$ (using the dominated convergence theorem). Thus, $\int_{Q_{T}} \psi A^{n} w^{n}$ converges to $\int_{Q_{T}} \psi A w$. In other words, $z^{n}=A^{n} w^{n}$ also converges weakly in $L^{2}\left(Q_{T}\right)$ to $z=A w$.

By (10), $\Delta \int_{0}^{t} z^{n}$ is bounded in $L^{2}\left(Q_{T}\right)$; since $\int_{0}^{t} z^{n}$ is bounded in $L^{2}\left(Q_{T}\right)$ as well, this implies that $\int_{0}^{t} z^{n}$ is bounded in $L^{2}\left(0, T ; H^{2}(\Omega)\right)$. We now may pass to the weak limit in (10) to deduce that $w$ is solution of (8).

For the uniqueness, let $w$ be the difference of two solutions of (8) (then $w(0)=0$ ). We denote $S(t)=\int_{0}^{t} A w$. Formally, the idea is to multiply the equation $w-\Delta S=0$ by $S^{\prime}=A w$. Then, after integration

$$
\int_{Q_{T}} A w^{2}=\int_{Q_{T}} S^{\prime} \Delta S=-\int_{Q_{T}} \nabla S^{\prime} \nabla S=-\int_{Q_{T}} \frac{1}{2} \partial_{t}|\nabla S(t)|^{2}=-\int_{\Omega} \frac{1}{2}|\nabla S(T)|^{2} \leq 0 .
$$

Whence $w \equiv 0$ since $A>0$.

Since we do not know whether $\nabla S^{\prime} \in L^{2}\left(Q_{T}\right)$, we have to justify this computation in an approximate way. For $h \in(0, T)$, let us denote

$$
\forall h \in(0, T), \quad S_{h}(t):=\frac{S(t+h)-S(t)}{h}=\frac{1}{h} \int_{t}^{t+h}(A w)(s) d s .
$$

Note that

$$
S_{h} \in L^{2}\left(0, T-h ; H^{2}(\Omega)\right),\left\|S_{h}-A w\right\|_{L^{2}\left(Q_{T-h}\right)} \rightarrow 0 \text { as } h \rightarrow 0 .
$$

We have

$$
\forall t \in[0, T-h), w(t+h)+w(t)-\Delta[S(t)+S(t+h)]=0 .
$$

We multiply by $S_{h}(t)$ and integrate over $\Omega$ to obtain

$$
\int_{\Omega}[w(t+h)+w(t)] S_{h}(t)=-\int_{\Omega} \nabla S_{h}(t)[\nabla S(t+h)+\nabla S(t)]=-\int_{\Omega} \frac{1}{h}\left\{|\nabla S(t+h)|^{2}-|\nabla S(t)|^{2}\right\} .
$$


After integration on $[0, T-h]$ and an easy change of variable, we have:

$$
\int_{Q_{T-h}}[w(\cdot+h)+w] S_{h}=-\frac{1}{h} \int_{(T-h, T) \times \Omega}|\nabla S|^{2}+\frac{1}{h} \int_{(0, h) \times \Omega}|\nabla S|^{2} \leq \frac{1}{h} \int_{Q_{h}}|\nabla S|^{2} .
$$

To pass to the limit as $h \rightarrow 0$, we use

$$
\int_{Q_{h}}|\nabla S|^{2}=\int_{Q_{h}}-S w=\int_{\Omega}-\int_{0}^{h}\left[w(t) \int_{0}^{t}(A w)(\sigma) d \sigma\right] d t \leq\|A\|_{L^{\infty}\left(Q_{T}\right)} h \int_{Q_{h}} w^{2} d t .
$$

Now, letting $h$ decrease to 0 in (16) and using that $S_{h} \rightarrow A w$ in $L^{2}(\operatorname{see}(15))$, lead to $\int_{Q_{T}} 2 w A w \leq 0$, whence $w \equiv 0$.

Let us now prove the continuity result. Let us first notice that, for any solution of (8), we have the identity

$$
\int_{Q_{T}} A w^{2}+\int_{\Omega} \frac{1}{2}\left|\nabla \int_{0}^{T} A w\right|^{2}=\int_{Q_{T}} w_{0} A w
$$

This may be justified as we did above for the uniqueness (namely in the case $w_{0}=0$ ) by passing to the limit in the following identity where $S(t)=\int_{0}^{t} A w, S_{h}(t)=[S(t+h)-S(t)] / h$ :

$$
\begin{gathered}
\int_{Q_{T-h}}[w(\cdot+h)+w] S_{h}+\nabla S_{h} \nabla[S(\cdot+h)+S]=2 \int_{Q_{T-h}} w_{0} S_{h}, \\
\int_{Q_{T-h}}[w(\cdot+h)+w] S_{h}+\frac{1}{h} \int_{(T-h, T) \times \Omega}|\nabla S|^{2}-\frac{1}{h} \int_{(0, h) \times \Omega}|\nabla S|^{2}=2 \int_{Q_{T-h}} w_{0} S_{h} .
\end{gathered}
$$

And we pass to the limit as above as $h \rightarrow 0$ to obtain (17) (at least a.e.T).

Let $w^{n}=w\left(A^{n}, w_{0}^{n}\right)$. As in the beginning of this proof (see (17), (13)), the relation

$$
\int_{Q_{T}} A^{n}\left(w^{n}\right)^{2}+\int_{\Omega} \frac{1}{2}\left|\nabla \int_{0}^{T} A^{n} w^{n}\right|^{2}=\int_{Q_{T}} w_{0}^{n} A^{n} w^{n} .
$$

proves that $w^{n}$ is bounded in $L^{2}\left(Q_{T}\right)$. From (10), we deduce that $\int_{0}^{t} A^{n} w^{n}$ is bounded in $L^{2}\left(0, T ; H^{2}(\Omega)\right)$. A subsequence of $\left(w^{n}, \Delta \int_{0}^{t} A^{n} w^{n}\right)$ converges weakly in $L^{2}\left(Q_{T}\right)^{2}$ to $\left(w, \Delta \int_{0}^{t} A w\right)$ and $w$ is solution of the limit problem (8). By uniqueness, the full sequence converges. Since $A^{n} \rightarrow A$ a.e., $\sqrt{A^{n}} w^{n}$ converges also weakly in $L^{2}\left(Q_{T}\right)$ to $\sqrt{A} w$ and, by the estimate $(20), \nabla \int_{0}^{T} A^{n} w^{n}$ converges weakly in $L^{2}(\Omega)$, the limit being necessarily $\nabla \int_{0}^{T} A w$. In particular

$$
\int_{Q_{T}} A w^{2} \leq \liminf _{n \rightarrow \infty} \int_{Q_{T}} A^{n}\left(w^{n}\right)^{2}, \int_{\Omega}\left|\nabla \int_{0}^{T} A w\right|^{2} \leq \liminf _{n \rightarrow \infty} \int_{\Omega}\left|\nabla \int_{0}^{T} A^{n} w^{n}\right|^{2} .
$$

But, since $\lim _{n \rightarrow \infty} \int_{Q_{T}} w_{0}^{n} A^{n} w^{n}=\int_{Q_{T}} w_{0} A w$, and since the identity (17) is true for $w$, it follows from (20), (17) that equality holds in the two inequalities (21). In particular, the norm of $\sqrt{A^{n}} w^{n}$ in $L^{2}\left(Q_{T}\right)$ converges to the norm of $\sqrt{A} w$; this implies that the $L^{2}\left(Q_{T}\right)$-weak convergence of $\sqrt{A^{n}} w^{n}$ to $\sqrt{A} w$ is actually strong. Using again the pointwise convergence of $A^{n}$, we deduce that $w^{n}$ converges strongly in $L^{2}\left(Q_{T}\right)$ as well. 
Remark 2.4 As a consequence of $(17)$, there is a constant $C=C\left(\underline{a}, \bar{a},\left\|w_{0}\right\|_{L^{2}(\Omega)}\right)$ such that for any solution $w$ of (8),

$$
\|w\|_{L^{2}\left(Q_{T}\right)} \leq \sqrt{T} C
$$

The next step is the definition of a compact continuous mapping $\mathcal{T}$ whose fixed points are solutions of (7). We introduce the Hilbert space

$$
X=\Pi_{1 \leq i \leq I} X_{i}, X_{i}=\left\{v \in L^{2}\left(Q_{T}\right): \partial_{t}\left(J_{\delta_{i}} v\right) \in L^{2}\left(Q_{T}\right)\right\}
$$

where the Hilbert norm $\|\cdot\|_{i}$ is defined on $X_{i}$ by

$$
\|v\|_{i}^{2}:=\|v\|_{L^{2}\left(Q_{T}\right)}^{2}+\left\|\partial_{t}\left(J_{\delta_{i}} v\right)\right\|_{L^{2}\left(Q_{T}\right)}^{2}
$$

and where $J_{\delta}=(I-\delta \Delta)^{-1}$ is the resolvent of the Laplace operator on $L^{2}(\Omega)$ with homogeneous Neumann boundary conditions, that is

$$
\left[f \in L^{2}(\Omega), Z=J_{\delta} f\right] \Leftrightarrow\left[Z \in H^{2}(\Omega), Z-\delta \Delta Z=f, \partial_{n} Z=0 \text { on } \partial \Omega\right] .
$$

Definition 2.5 We fix $u^{0} \in L^{2}(\Omega,[0, \infty))^{I}$. Let $v=\left(v_{1}, \ldots, v_{I}\right) \in X$ and let $\tilde{u}=\left(\tilde{u}_{1}, \ldots, \tilde{u}_{I}\right)$ be the solution of (see [6], Proposition 9.24 and Theorem 9.26):

$$
\forall i=1, \ldots, I, \tilde{u}_{i} \in L^{2}\left(0, T ; H^{2}(\Omega)\right), \tilde{u}_{i}-\delta_{i} \Delta \tilde{u}_{i}=v_{i} \text { on } Q_{T}, \quad \partial_{n} \tilde{u}_{i}=0 \text { on } \Sigma_{T} .
$$

Next, we define

$$
\mathcal{T}: X \rightarrow X \text { by } \mathcal{T}(v):=u=\left(u_{1}, \ldots, u_{I}\right),
$$

where $u_{i}$ is the solution $w$ of $(8)$ with $A=a_{i}\left([\tilde{u}]^{+}\right), w_{0}=u_{i}^{0} ;[\tilde{u}]^{+}=\left(\left[\tilde{u}_{1}\right]^{+}, \ldots,\left[\tilde{u}_{I}\right]^{+}\right)$and $\left[\tilde{u}_{i}\right]^{+}$is the positive part of $\tilde{u}_{i}$.

Proposition 2.6 Assume (3), (6) and $\forall i=1, \ldots, I, u_{i}^{0} \in L^{2}(\Omega ;[0, \infty))$. Then the mapping $\mathcal{T}$ is continuous and compact from $X$ into itself.

Proof of Proposition 2.6: First, remark that for $v \in X, u=\mathcal{T}(v) \in X$. Indeed, since $u_{i}$ is solution of (8) with $A=a_{i}\left([\tilde{u}]^{+}\right)$and $w_{0}=u_{i}^{0}$, we may write

$$
J_{\delta_{i}} u_{i}=J_{\delta_{i}} \Delta \int_{0}^{t} A u_{i}+J_{\delta_{i}} u_{i}^{0}=\int_{0}^{t} \Delta J_{\delta_{i}}\left(A u_{i}\right)+J_{\delta_{i}} u_{i}^{0} \Rightarrow \partial_{t}\left(J_{\delta_{i}} u_{i}\right)=\Delta J_{\delta_{i}}\left(A u_{i}\right) \in L^{2}\left(Q_{T}\right) .
$$

Let $v^{n}$ be a bounded sequence in $X$. Up to a subsequence, me may assume that $v_{i}^{n}$ converges weakly to $v_{i}$ in $L^{2}\left(Q_{T}\right)$. Then

$$
\tilde{u}_{i}^{n}-\delta_{i} \Delta \tilde{u}_{i}^{n}=v_{i}^{n} \text { on } Q_{T}, \partial_{n} \tilde{u}_{i}^{n}=0 \text { on } \Sigma_{T} \Rightarrow \partial_{t} \tilde{u}_{i}^{n}=\partial_{t}\left(J_{\delta_{i}} v_{i}^{n}\right)
$$

Thus $\tilde{u}_{i}^{n}$ is bounded in $L^{2}\left(0, T ; H^{2}(\Omega)\right)$ and $\partial_{t} \tilde{u}_{i}^{n}=\partial_{t}\left(J_{\delta_{i}} v_{i}^{n}\right)=J_{\delta_{i}}\left(\partial_{t} v_{i}^{n}\right)$ is bounded in $L^{2}\left(Q_{T}\right)$. As a consequence, $\tilde{u}_{i}^{n}$ is relatively compact in $L^{2}\left(Q_{T}\right)$, and so is $\left[\tilde{u}_{i}^{n}\right]^{+}$. Up to a subsequence again, we may assume that they converge strongly in $L^{2}\left(Q_{T}\right)$ and a.e. in $Q_{T}$. By continuity of $a_{i}, a_{i}\left(\left[\tilde{u}^{n}\right]^{+}\right)$ 
converges a.e. and $0<\underline{d} \leq a_{i}\left(\left[\tilde{u}^{n}\right]^{+}\right) \leq \bar{d}<\infty$. By Lemma 2.3, $u^{n}:=\mathcal{T}\left(v^{n}\right)$ converges (up to a subsequence) strongly in $L^{2}\left(Q_{T}\right)$. Moreover

$$
u_{i}^{n}=\Delta\left(\int_{0}^{t} a_{i}\left(\left[\tilde{u}^{n}\right]^{+}\right) u_{i}^{n}\right)+u_{i}^{0} \Rightarrow \partial_{t}\left(J_{\delta_{i}} u_{i}^{n}\right)=\Delta J_{\delta_{i}}\left[\left(a_{i}\left[\tilde{u}^{n}\right]^{+}\right) u_{i}^{n}\right] .
$$

But the Yosida approximation $\Delta J_{\delta_{i}}$ is Lipschitz continuous on $L^{2}\left(Q_{T}\right)$, and $a_{i}\left(\left[\tilde{u}^{n}\right]^{+}\right) u_{i}^{n}$ converges in $L^{2}\left(Q_{T}\right)$. Therefore, $\partial_{t}\left(J_{\delta_{i}} u_{i}^{n}\right)$ converges also in $L^{2}\left(Q_{T}\right)$. Finally, this proves that $u^{n}$ converges in $X$ (at least up to a subsequence), whence the compactness of $\mathcal{T}$.

For the continuity of $\mathcal{T}$, let $v^{n} \rightarrow v$ in $X$ as $n \rightarrow \infty$. If $\tilde{u}^{n}=\left(\tilde{u}_{1}^{n}, \ldots, \tilde{u}_{I}^{n}\right)$ is the solution of

$$
\forall i=1, \ldots, I, \quad \tilde{u}_{i}^{n}-\delta_{i} \Delta \tilde{u}_{i}^{n}=v_{i}^{n} \text { on } Q_{T}, \partial_{n} \tilde{u}_{i}^{n}=0 \text { on } \Sigma_{T}
$$

then $\tilde{u}_{i}^{n}$ converges in $L^{2}\left(0, T ; H^{2}(\Omega)\right)$ to the solution $\tilde{u}_{i}$ of

$$
\tilde{u}_{i}-\delta_{i} \Delta \tilde{u}_{i}=v_{i} \text { on } Q_{T}, \partial_{n} \tilde{u}_{i}=0 \text { on } \Sigma_{T} .
$$

By definition, $u^{n}=\mathcal{T}\left(v^{n}\right)=\left(u_{1}^{n}, \ldots, u_{I}^{n}\right)$ is the solution of

$$
\left\{\begin{array}{l}
u_{i}^{n} \in L^{2}\left(Q_{T}\right), \int_{0}^{t} a_{i}\left(\left[\tilde{u}^{n}\right]^{+}\right) u_{i}^{n} \in L^{2}\left(0, T ; H^{2}(\Omega)\right), \\
u_{i}^{n}-\Delta\left(\int_{0}^{t} a_{i}\left(\left[\tilde{u}^{n}\right]^{+}\right) u_{i}^{n}\right)=u_{i}^{0} \text { on } Q_{T}, \partial_{n}\left(\int_{0}^{t} a_{i}\left(\left[\tilde{u}^{n}\right]^{+}\right) u_{i}^{n}\right)=0 \text { on } \Sigma_{T} .
\end{array}\right.
$$

Using the compactness of $\mathcal{T}$ proven above, the sequence $\left(u^{n}\right)_{n \in \mathbb{N}}$ is relatively compact in $X$. Let $u^{\infty}=\lim _{p \rightarrow \infty} u^{n_{p}}$ be a limit point. Up to a subsequence, $\tilde{u}_{i}^{n_{p}}$ converges a.e. to $\tilde{u}_{i}$. By continuity of $a_{i}, a_{i}\left(\left[\tilde{u}^{n_{p}}\right]^{+}\right) \rightarrow A_{i}:=a_{i}\left([\tilde{u}]^{+}\right)$almost everywhere, and it is uniformly bounded from above and from below. According to Lemma 2.3, we can pass to the limit as $n_{p} \rightarrow+\infty$ in (24). By the uniqueness result in Lemma 2.3 with $A=A_{i}$, we necessarily have $u^{\infty}=\mathcal{T}(v)$. The sequence $\left(u^{n}\right)_{n \in \mathbb{N}}$ lies in a compact set and has a unique possible limit point, so $u^{n}=\mathcal{T}\left(v^{n}\right) \rightarrow \mathcal{T}(v)$ and $\mathcal{T}$ is continuous on $X$.

Proof of Proposition 2.1: Let $T \in(0, \infty)$ and $\sigma \in[0,1]$. Suppose that $u \in X$ is a solution of $u=\sigma \mathcal{T}(u)$. By definition of $\mathcal{T}$, we have

$$
\left\{\begin{array}{l}
\forall i=1, \ldots, I, u_{i} \in L^{2}\left(Q_{T}\right), u_{i} \geq 0 \\
\tilde{u}_{i}, \int_{0}^{t} a_{i}(\tilde{u}) u_{i} \in L^{2}\left(0, T ; H^{2}(\Omega)\right) \\
\tilde{u}_{i}-\delta_{i} \Delta \tilde{u}_{i}=u_{i} \text { on } Q_{T}, \partial_{n} \tilde{u}_{i}=0 \text { on } \Sigma_{T} \\
u_{i}-\Delta \int_{0}^{t} a_{i}(\tilde{u}) u_{i}=\sigma u_{i}^{0} \text { on } Q_{T}, \partial_{n}\left(\int_{0}^{t} a_{i}(\tilde{u}) u_{i}\right)=0 \text { on } \Sigma_{T}
\end{array}\right.
$$

The initial conditions $\sigma u_{i}^{0}$ are uniformly bounded in $L^{2}(\Omega)$ for $\sigma \in[0,1]$. Therefore, by the estimate (22), the function $u_{i}$ remains bounded in $L^{2}\left(Q_{T}\right)$, independently of $\sigma$. We also have $\partial_{t}\left(J_{\delta_{i}} u_{i}\right)=$ $\Delta J_{\delta_{i}}\left(a_{i}(\tilde{u}) u_{i}\right)$, so $u$ is bounded in $X$ independently of $\sigma$. Using Proposition 2.6 and Leray-Schauder's Lemma 2.2, we can conclude that $\mathcal{T}$ has a fixed point, which is a nonnegative solution of (7) (the nonnegativity of $\tilde{u}_{i}$ is a consequence of $u_{i} \geq 0$ and of the maximum principle property of $\left(I-\delta_{i} \Delta\right)^{-1}$ with homogeneous Neumann boundary conditions, see e.g. [6], Proposition 9.30).

\section{$3 \quad L^{\infty}$-estimate of $\tilde{u}$ in Proposition 2.1}

A main estimate in the proof of Theorem 1.1 is given in the next proposition. 
Proposition 3.1 Assume $u^{0} \in L^{\infty}(\Omega,[0,+\infty))^{I}$ and (3), (6) as in Proposition 2.1. Let us define

$$
\forall k \geq 0, \quad G(k)=\max _{i}\left\{\sup _{r \in[0, k]^{I}} a_{i}(r)\right\} .
$$

Then, for any solution $u, \tilde{u}$ of Proposition 2.1, we have

$$
\max _{1 \leq i \leq I}\left\{\delta_{i}\left\|\tilde{u}_{i}\right\|_{L^{\infty}\left(Q_{T}\right)}+\left\|\int_{0}^{t} a_{i}(\tilde{u}) u_{i}\right\|_{L^{\infty}\left(Q_{T}\right)}\right\} \leq M_{0}+M_{1} T G\left(k_{0}\right)
$$

where $M_{0}, M_{1}$ and $k_{0}$ depend only on $u^{0}, \underline{\delta}:=\min _{i} \delta_{i}, \bar{\delta}:=\max _{i} \delta_{i}$.

The proof of Proposition 3.1 uses the following classical lemma.

Lemma 3.2 Let $f \in L^{\infty}(\Omega)$ and let $w$ satisfy

$$
w \in H^{2}(\Omega), w \geq 0,-\Delta w \leq f \text { on } \Omega, \partial_{n} w=0 \text { on } \partial \Omega .
$$

Then there exists $C=C(\Omega)$ such that

$$
\|w\|_{L^{\infty}(\Omega)} \leq C\left(\|f\|_{L^{\infty}(\Omega)}+\int_{\Omega} w\right) .
$$

Proof: First, we rewrite the equation as $w-\Delta w \leq f+w$. Let us fix $p \in(N / 2, \infty)$. Using $w \geq 0$, the comparison principle and elliptic regularity theory, we know (see e.g. [9], Theorem 8.15) the existence of $C=C(\Omega, p)$ such that

$$
\begin{aligned}
\|w\|_{L^{\infty}} & \leq C\left(\|f+w\|_{L^{p}}\right) \leq C\left(\|f\|_{L^{p}}+\|w\|_{L^{p}}\right) \\
& \leq C\left(\|f\|_{L^{p}}+\|w\|_{L^{\infty}}^{(p-1) / p}\left(\int_{\Omega} w\right)^{1 / p}\right) \\
& \leq C\left(\|f\|_{L^{p}}+\varepsilon\|w\|_{L^{\infty}}+c(\varepsilon) \int_{\Omega} w\right) \text { (Young's inequality) }
\end{aligned}
$$

and we conclude choosing $\varepsilon$ small enough.

Remark 3.3 Obviously, the conclusion of Lemma 3.2 would be the same when assuming only $f \in$ $L^{p}(\Omega), p>N / 2$.

Proof of Proposition 3.1: We rewrite the equations in $u_{i}, \tilde{u}_{i}$ of Proposition 2.1 as

$$
\tilde{u}_{i}-\Delta\left(\delta_{i} \tilde{u}_{i}+\int_{0}^{t} a_{i}(\tilde{u}) u_{i}\right)=u_{i}^{0}, \quad \tilde{u}_{i}-\Delta w_{i}=u_{i}^{0}, \quad w_{i}=\delta_{i} \tilde{u}_{i}+\int_{0}^{t} a_{i}(\tilde{u}) u_{i} .
$$

We sum up the equations (29), denoting $\tilde{U}=\sum_{i} \tilde{u}_{i}, W=\sum_{i} w_{i}$ :

$$
\tilde{U}-\Delta W=U^{0}:=\sum_{i} u_{i}^{0}
$$

Next, we apply Lemma 3.2 with $w=W(t)$, a.e.t, $f=U^{0}$ (note that $-\Delta W(t) \leq U^{0}$ ). It gives

$$
\text { a.e.t, }\|W(t)\|_{L^{\infty}(\Omega)} \leq C\left(\left\|U^{0}\right\|_{L^{\infty}(\Omega)}+\int_{\Omega} W(t)\right) \text {. }
$$


By nonnegativity of $\tilde{u}_{i}, a_{i}(\tilde{u}) u_{i}$, we also have (see the definitions of $\left.W, w_{i}\right): \forall i=1, \ldots, I$, a.e.t $\in[0, T]$ :

$$
\delta_{i}\left\|\tilde{u}_{i}(t)\right\|_{L^{\infty}(\Omega)},\left\|\int_{0}^{t} a_{i}(\tilde{u}) u_{i}\right\|_{L^{\infty}(\Omega)} \leq\|W(t)\|_{L^{\infty}(\Omega)} .
$$

Then, to end the proof of Proposition 3.1, it is sufficient to prove the following lemma.

\section{Lemma 3.4}

$$
\text { a.e.t } \in[0, T], \int_{\Omega} W(t) \leq C_{0}+C_{1} T G\left(k_{0}\right),
$$

where $C_{0}, C_{1}, k_{0}$ depend only on $u^{0}, \underline{\delta}, \bar{\delta}$ and $G$ is defined in (26).

Proof of Lemma 3.4: By integrating the equations on $u_{i}$ and $\tilde{u}_{i}$ in Proposition 2.1, we get:

$$
\forall t \geq 0, \quad \int_{\Omega} u_{i}(t)=\int_{\Omega} \tilde{u}_{i}(t)=\int_{\Omega} u_{i}^{0} .
$$

Recall that $\tilde{u}_{i}, w_{i} \in L^{2}\left(0, T ; H^{2}(\Omega)\right), a_{i}(\tilde{u}) u_{i} \in L^{2}\left(Q_{T}\right)$. We also have $\partial_{t} \tilde{u}_{i}=\Delta J_{\delta_{i}}\left(a_{i}(\tilde{u}) u_{i}\right) \in L^{2}\left(Q_{T}\right)$ From (29), we may write with $\partial_{t} w_{i}=\delta_{i} \partial_{t} \tilde{u}_{i}+a_{i}(\tilde{u}) u_{i} \in L^{2}\left(Q_{T}\right)$

$$
\partial_{t} w_{i}-\delta_{i} \Delta\left(\partial_{t} w_{i}\right)=a_{i}(\tilde{u}) u_{i}
$$

Differentiating $\partial_{n} w_{i}=0$ with respect to $t$ on $\partial \Omega$ leads formally to $\partial_{n}\left(\partial_{t} w_{i}\right)=0$. Let us check that $\partial_{t} w_{i}=\theta(t)$ where $\theta(t)$ is the unique solution of

$$
\theta \in L^{2}\left(0, T ; H^{2}(\Omega)\right) \text {, a.e.t } \in[0, T], \theta(t)-\delta_{i} \Delta \theta(t)=\left(a_{i}(\tilde{u}) u_{i}\right)(t), \partial_{n} \theta(t)=0 \text { on } \partial \Omega .
$$

Using also $a_{i}(\tilde{u}) u_{i} \geq 0$, it will then follow that

$$
\partial_{t} w_{i} \geq 0, \quad \partial_{t} w_{i} \in L^{2}\left(0, T ; H^{2}(\Omega)\right),\left\|\partial_{t} w_{i}\right\|_{L^{2}\left(Q_{T}\right)} \leq\left\|a_{i}(\tilde{u}) u_{i}\right\|_{L^{2}\left(Q_{T}\right)} .
$$

By integration in time of (34), and with $\Theta(t)=\int_{0}^{t} \theta(s) d s$, we have

$$
\Theta(t)-\delta_{i} \Delta \Theta(t)=\int_{0}^{t}\left(a_{i}(\tilde{u}) u_{i}\right)(t), \partial_{n} \Theta(t)=0 \text { on } \partial \Omega
$$

Comparing with $w_{i}-\delta_{i} \Delta w_{i}=\delta_{i} u_{i}^{0}+\int_{0}^{t} a_{i}(\tilde{u}) u_{i}, \partial_{n} w_{i}=0$ implies by uniqueness that: $\Theta(t)=w_{i}+\left(I-\delta_{i} \Delta\right)^{-1} u_{i}^{0}$, whence $\Theta^{\prime}(t)=\theta=w_{i}$ after differentiating in $t$.

We denote

$$
\widetilde{V}=\sum_{i} \delta_{i} \widetilde{u}_{i}, B=\sum_{i} a_{i}(\tilde{u}) u_{i}
$$

Recall also that

$$
\widetilde{U}=\sum_{i} \tilde{u}_{i}, W=\sum_{i} w_{i}, w_{i}=\delta_{i} \tilde{u}_{i}+\int_{0}^{t} a_{i}(\tilde{u}) u_{i} .
$$

Summing the $I$ equations in $u_{i}, \tilde{u}_{i}$ as in (30), we have

$$
\bar{\delta}^{-1} \widetilde{V}-\Delta W \leq \widetilde{U}-\Delta W=U^{0} .
$$


We multiply this equation by $\partial_{t} W=\sum_{i} \partial_{t} w_{i}=\partial_{t} \widetilde{V}+B \geq 0$ (see (35)) and we get

$$
\bar{\delta}^{-1} \int_{\Omega} \widetilde{V}\left(\partial_{t} \widetilde{V}+B\right)+\frac{1}{2} \int_{\Omega} \partial_{t}|\nabla W|^{2} \leq \int_{\Omega} U^{0}\left(\partial_{t} \widetilde{V}+B\right)
$$

We integrate in time to obtain (we denote $\widetilde{V}^{0}:=\widetilde{V}(0)=W(0)$ )

$$
\int_{\Omega} \widetilde{V}^{2}(T)+\int_{Q_{T}} 2 B \widetilde{V}+\bar{\delta} \int_{\Omega}|\nabla W(T)|^{2} \leq \int_{\Omega}\left(\widetilde{V}^{0}\right)^{2}+\bar{\delta}\left|\nabla \widetilde{V}^{0}\right|^{2}+2 \bar{\delta} U^{0}\left(\widetilde{V}(T)-\widetilde{V}^{0}\right)+\int_{Q_{T}} 2 \bar{\delta} U^{0} B
$$

Since we have by definition

$$
\bar{\delta} U^{0}=\bar{\delta} \widetilde{U}^{0}-\bar{\delta} \Delta \widetilde{V}^{0} \geq \widetilde{V}^{0}-\bar{\delta} \Delta \widetilde{V}^{0}
$$

we have

$$
\int_{\Omega}\left(\widetilde{V}^{0}\right)^{2}+\bar{\delta}\left|\nabla \widetilde{V}^{0}\right|^{2}-2 \bar{\delta} U^{0} \widetilde{V}^{0} \leq-\int_{\Omega}\left(\widetilde{V}^{0}\right)^{2}+\bar{\delta}\left|\nabla \widetilde{V}^{0}\right|^{2} \leq 0
$$

So that (37) becomes,

$$
\int_{\Omega} \widetilde{V}^{2}(T)+\int_{Q_{T}} 2 B \widetilde{V}+\bar{\delta} \int_{\Omega}|\nabla W(T)|^{2} \leq 2 \bar{\delta} \int_{\Omega} U^{0} \widetilde{V}(T)+\int_{Q_{T}} 2 \bar{\delta} U^{0} B .
$$

We have in particular, with $\left\|U^{0}\right\|_{\infty}=\left\|U^{0}\right\|_{L^{\infty}(\Omega)}$, and by using (32):

$$
\int_{Q_{T}} B \widetilde{V} \leq \bar{\delta}\left\|U^{0}\right\|_{\infty}\left(\int_{\Omega} \widetilde{V}^{0}+\int_{Q_{T}} B\right)
$$

Thus, we have for any $k>0$

$$
k \int_{Q_{T} \cap\{\widetilde{V} \geq k\}} B \leq \bar{\delta}\left\|U^{0}\right\|_{\infty}\left(\int_{\Omega} \widetilde{V}^{0}+\int_{Q_{T} \cap\{\widetilde{V}<k\}} B+\int_{Q_{T} \cap\{\widetilde{V} \geq k\}} B\right) .
$$

Note that, $\{\widetilde{V}<k\} \subset \cap_{i}\left\{\tilde{u}_{i} \leq k \underline{\delta}^{-1}\right\}$. Thanks to the $L^{1}$ estimate (32), we have

$$
\int_{Q_{T} \cap\{\tilde{V}<k\}} B=\int_{Q_{T} \cap\{\tilde{V}<k\}} \sum_{i} a_{i}(\tilde{u}) u_{i} \leq T\left[\int_{\Omega} U^{0}\right] G\left(k \underline{\delta}^{-1}\right),
$$

where $G$ is defined in (26). Finally choosing $k=2 \bar{\delta}\left\|U^{0}\right\|_{\infty}$ in (40), we obtain

$$
\int_{Q_{T} \cap\{\widetilde{V} \geq k\}} B \leq\left(2 \int_{\Omega} \widetilde{V}^{0}+T\left[\int_{\Omega} U^{0}\right] G\left(k_{0}\right)\right), \quad k_{0}=2 \underline{\delta}^{-1}\left(\bar{\delta}\left\|U^{0}\right\|_{\infty}\right) .
$$

Adding the two last inequalities gives

$$
\int_{Q_{T}} B \leq C_{0}+C_{1} T G\left(k_{0}\right)
$$

where $C_{1}$ depends only on $u^{0}, \bar{\delta}, \underline{\delta}$.

To end the proof of Lemma 3.4, we use that $W(t)=\sum_{i} \delta_{i} \tilde{u}_{i}(t)+\int_{0}^{t} B(s) d s$ so that

$$
\forall t \in[0, T], \int_{\Omega} W(t) \leq \int_{\Omega} \widetilde{U}^{0}+\int_{Q_{T}} B
$$


From the $L^{\infty}$-estimate of Proposition 3.1, we may now deduce that the problem (4) in Theorem 1.1 has at least a weak solution under the only assumption of continuity of the $a_{i}$ 's.

Corollary 3.5 Assume (3) (only) and $\forall i=1, \ldots, I, u_{i}^{0} \in L^{\infty}(\Omega ;[0, \infty))$. Then, there exists a nonnegative solution $u=\left(u_{1}, \ldots, u_{I}\right)$ to the system

$$
\left\{\begin{array}{l}
\forall T>0, \forall i=1, \ldots, I, \\
u_{i}, a_{i}(\tilde{u}) u_{i} \in L^{2}\left(Q_{T}\right), \int_{0}^{t} a_{i}(\tilde{u}) u_{i} \in L^{2}\left(0, T ; H^{2}(\Omega)\right) \\
\tilde{u}_{i} \in L^{\infty}\left(Q_{T}\right) \cap L^{2}\left(0, T ; H^{2}(\Omega)\right), \tilde{u}_{i}-\delta_{i} \Delta \tilde{u}_{i}=u_{i} \text { on } Q_{T} \\
u_{i}-\Delta\left(\int_{0}^{t} a_{i}(\tilde{u}) u_{i}\right)=u_{i}^{0} \text { on } Q_{T} \\
\partial_{n} \tilde{u}_{i}=0=\partial_{n}\left(\int_{0}^{t} a_{i}(\tilde{u}) u_{i}\right) \text { on } \Sigma_{T} .
\end{array}\right.
$$

Proof: Here, $a_{i}$ is assumed to satisfy only (3) (and not (6)). Let $T>0$. We introduce $M_{2}:=$ $\underline{\delta}^{-1}\left[M_{0}+M_{1} T G\left(k_{0}\right)\right]$ where the function $G$ is defined in (26) of Proposition 3.1 and $M_{0}, M_{1}, k_{0}$ are defined in (27) of the same proposition. We define

$$
\forall r \in\left[0, M_{2}\right]^{I}, \quad \bar{a}_{i}(r):=a_{i}(r), \forall r \in[0, \infty)^{I} \backslash\left[0, M_{2}\right]^{I}, \bar{a}_{i}(r)=\min \left\{a_{i}(r), G\left(M_{2}\right)\right\} .
$$

Then, $\bar{a}_{i}$ is continuous on $[0, \infty)^{I}$ and

$$
0<\underline{d} \leq \bar{a}_{i} \leq G\left(M_{2}\right)<\infty, \quad \bar{a}_{i} \leq a_{i} .
$$

Therefore, we may apply Proposition 2.1 with $a_{i}$ replaced by $\bar{a}_{i}$. By Proposition 3.1 , the corresponding $\tilde{u}$ satisfies

$$
\forall i=1, . ., I,\left\|\tilde{u}_{i}\right\|_{L^{\infty}\left(Q_{T}\right)} \leq \underline{\delta}^{-1}\left[M_{0}+M_{1} T \bar{G}\left(k_{0}\right)\right],
$$

where $\bar{G}$ is defined as in (26) with $a_{i}$ replaced by $\bar{a}_{i}$. But $\bar{G}\left(k_{0}\right) \leq G\left(k_{0}\right)$, so that

$$
\forall i=1, \ldots, I, 0 \leq \tilde{u}_{i} \leq M_{2}, \bar{a}_{i}(\tilde{u})=a_{i}(\tilde{u}) .
$$

Therefore, the solution obtained with the data $\bar{a}_{i}$ is also solution with the data $a_{i}$.

This provides a solution of (42) in Corollary 3.5 with the estimate (27), but only on $[0, T]$ and it may depend on $T$. To construct a global solution on $(0, \infty)$, we may argue as follows: let $T_{p}$ be an increasing sequence of times with $\lim _{p \rightarrow+\infty} T_{p}=+\infty$. Let $u^{p}$ be a solution of our problem on the interval $\left[0, T_{p}\right]$ given by the above proof. For $k \in \mathbb{N}$, we denote by $X^{k}$ the space $X$ as defined in (23) with $T$ replaced by $T_{k}$ and we denote by $\mathcal{T}^{k}: X^{k} \rightarrow X^{k}$ the operator $\mathcal{T}$ with $T=T_{k}$. For $p \geq k$, we denote $u^{p, k}:=u_{\left[0, T_{k}\right]}^{p}$ so that $\mathcal{T}^{k}\left(u^{p, k}\right)=u^{p, k}$. We will prove that

$$
\forall k \in \mathbb{N},\left(u^{p, k}\right)_{p \geq k} \text { is relatively compact in } X^{k} .
$$

Thus, using a diagonal process, we obtain a sequence $p_{m} \rightarrow \infty$ as $m \rightarrow \infty$ and some limit $u$ defined on $(0, \infty)$ so that, for all $k \in \mathbb{N}, u^{p_{m}, k}$ converges to $u_{\left[0, T_{k}\right]}$ in $X^{k}$ as $m \rightarrow \infty$. Then, $\mathcal{T}_{k}\left(u_{\left[0, T_{k}\right]}\right)=u_{\left[0, T_{k}\right]}$ and $u$ is a global solution of (42).

Let $k$ be fixed in $\mathbb{N}$ and let us prove (43). By the $L^{\infty}$-estimate (27) in Proposition 3.1,

$$
\forall p \geq k, \quad\left\|\tilde{u}_{i}^{p}\right\|_{L^{\infty}\left(Q_{T_{k}}\right)} \leq \frac{1}{\delta_{i}}\left[M_{0}+M_{1} T_{k} G\left(k_{0}\right)\right] .
$$

Thus, $a_{i}\left(\tilde{u}^{p}\right)$ is uniformly bounded on $Q_{T_{k}}$. This implies by (22) that $u^{p}$ is bounded in $L^{2}\left(Q_{T_{k}}\right)^{I}$ and so is $\partial_{t} \tilde{u}^{p}$ since by $(35)$

$$
\delta_{i}\left\|\partial_{t} \tilde{u}_{i}^{p}\right\|_{L^{2}\left(Q_{T_{k}}\right)} \leq 2\left\|a_{i}\left(\tilde{u}^{p}\right) u_{i}^{p}\right\|_{L^{2}\left(Q_{T_{k}}\right)} \leq C(k) .
$$

Thus, $u^{p, k}$ is bounded in $X^{k}$ and, by compactness of $\mathcal{T}^{k}$, it is relatively compact in $X^{k}$, whence (43). 


\section{Proof of existence in Theorem 1.1}

Existence of a weak solution to (4) is already proved in Corollary 3.5. It only remains to prove that this solution is actually as regular as stated in Theorem 1.1. This will mainly be a consequence of the $L^{\infty}$-estimate on $\tilde{u}$ proved in the previous section, namely

$$
\forall i=1, \ldots, I,\left\|\tilde{u}_{i}\right\|_{L^{\infty}\left(Q_{T}\right)} \leq C_{0}+C_{1} T,\left\|a_{i}(\tilde{u})\right\|_{L^{\infty}\left(Q_{T}\right)} \leq C(T),
$$

where $C_{0}, C_{1}$ depend only on the data and $C(T)=G\left(C_{0}+C_{1} T\right)$.

We begin by the following simple estimates.

Proposition 4.1 Let $w_{i}=\delta_{i} \tilde{u}_{i}+\int_{0}^{t} a_{i}(\tilde{u}) u_{i}$ where $u, \tilde{u}$ is solution of (42) in Corollary 3.5. Assume $u^{0} \in L^{\infty}(\Omega,[0, \infty))^{I}$. Then,

$$
\forall T>0, \nabla w_{i} \in L^{\infty}\left(Q_{T}\right)^{N}, w_{i}, \partial_{t} w_{i} \in L^{\infty}\left(Q_{T}\right), \partial_{t} w_{i} \geq 0 .
$$

Proof: The fact that $w_{i} \in L^{\infty}\left(Q_{T}\right)$ is a consequence of (31) and Lemma 3.4. We recall the two equations (see (29), (33)):

$$
\tilde{u}_{i}-\Delta w_{i}=u_{i}^{0}, \partial_{t} w_{i}-\delta_{i} \Delta\left(\partial_{t} w_{i}\right)=a_{i}(\tilde{u}) u_{i}
$$

Since $w_{i}, \Delta w_{i} \in L^{\infty}\left(Q_{T}\right)$ and $\partial_{n} w_{i}=0$ on $\Sigma_{T}$, we deduce that $\nabla w_{i} \in L^{\infty}\left(Q_{T}\right)^{N}$ (at least). We have already seen that $\partial_{t} w_{i} \geq 0$ comes directly from the second equation and the nonnegativity of $a_{i}(\tilde{u}) u_{i}$. Now we rewrite this equation as

$$
\left(\partial_{t} w_{i}-C(T) \tilde{u}_{i}\right)-\delta_{i} \Delta\left(\partial_{t} w_{i}-C(T) \tilde{u}_{i}\right)=\left(a_{i}(\tilde{u})-C(T)\right) u_{i} \leq 0 .
$$

Together with $\partial_{n}\left(\partial_{t} w_{i}-C(T) \tilde{u}_{i}\right)=0$ on $\Sigma_{T}$, this implies

$$
\partial_{t} w_{i}-C(T) \tilde{u}_{i} \leq 0, \text { so that } 0 \leq \partial_{t} w_{i} \leq C(T)\left[C_{0}+C_{1} T\right]
$$

We will now prove that $U_{i}(t, x):=\int_{0}^{t}\left[a_{i}(\tilde{u}) u_{i}\right](s, x) d s$ is in $C^{\alpha}\left(\overline{Q_{T}}\right)$ so that, since $\tilde{u}_{i}=w_{i}-U_{i}$, it will follow that $\tilde{u}_{i}$ is not only bounded, but Hölder-continuous (at least).

To prove it, we rely on the $C^{\alpha}$-regularity theory of Krylov-Safonov for the solutions of nondivergence parabolic equations with bounded coefficients. We actually use them in the rather particular case of the operator $-A \Delta$ where $A$ is bounded from above and from below. We may state the result we need as follows:

Lemma 4.2 Let $A \in C\left(\overline{Q_{T}}\right), g \in L^{\infty}\left(Q_{T}\right), \underline{a}, \bar{a} \in(0, \infty)$ with $0<\underline{a} \leq A \leq \bar{a}<\infty$. Let $w \in$ $C^{2,1}\left(Q_{T}\right) \cap C^{1,1}\left(\overline{Q_{T}}\right)$ solution of

$$
\left\{\begin{array}{l}
\partial_{t} w-A \Delta w=g \text { in } Q_{T} \\
\partial_{n} w=0 \text { on } \Sigma_{T}, w(0)=0 .
\end{array}\right.
$$

Then, there exists $\alpha \in(0,1), C>0$ such that

$$
\|w\|_{T}^{(\alpha)} \leq C
$$

where $\alpha, C$ depend only on $\underline{a}, \bar{a}, T,\|g\|_{L^{\infty}\left(Q_{T}\right)}, \Omega$. 
Remark 4.3 Note that the $L^{\infty}$ estimate of $w$ is easy by a comparison argument (valid here thanks to the a priori regularity of $w$ and of $A$ ): we remark that the function $W(t, x):=t \sup g$ is a supersolution of the problem (46), so that $W \geq w$. Doing the same from below, we obtain

$$
\|w\|_{L^{\infty}\left(Q_{T}\right)} \leq T\|g\|_{L^{\infty}\left(Q_{T}\right)} .
$$

Next, we may use the Krylov-Safonov result: the global estimate with homogeneous Neumann boundary conditions as stated above may, for instance, be found in [10], Lemma 2.2 (in a quite more general setting). We more generally refer to [13], [10], [20] for this kind of results.

We apply this result to prove the regularity of $U_{i}=\int_{0}^{t} a_{i}(\tilde{u}) u_{i}$.

Proposition 4.4 Let $T>0$ and $u^{0} \in L^{\infty}(\Omega,[0, \infty))^{I}$. There exists $\alpha \in(0,1), C>0$ such that

$$
\left\|U_{i}\right\|_{T}^{(\alpha)}+\left\|\tilde{u}_{i}\right\|_{T}^{(\alpha)} \leq C
$$

Proof: Let $u, \tilde{u}$ be the solution of (42) in Corollary 3.5. Recall that $0<\underline{d} \leq a_{i}(\tilde{u}) \leq C(T)$. Since Lemma 4.2 a priori applies to regular solutions only, we will use a convenient approximation of $u$. For this, let $A^{n}$ be a smooth approximation of $a_{i}(\tilde{u})$ such that

$$
0<\underline{d} \leq A^{n} \leq C(T), \quad A^{n} \rightarrow a_{i}(\tilde{u}) \text { a.e. }
$$

Let also $v^{n}$ be a smooth approximation of $u_{i}^{0}$ such that

$$
0 \leq v^{n} \leq\left\|u_{i}^{0}\right\|_{L^{\infty}(\Omega)}, \quad v^{n} \rightarrow u_{i}^{0} \text { in } L^{2}(\Omega) .
$$

Let $u_{i}^{n}$ be the solution of

$$
\partial_{t} u_{i}^{n}-\Delta\left(A^{n} u_{i}^{n}\right)=0, \partial_{n} u_{i}^{n}=0 \text { on } \Sigma_{T}, u_{i}^{n}(0)=v^{n} .
$$

Then, after integration in time, we see that $U_{i}^{n}=\int_{0}^{t} A^{n} u_{i}^{n}$ satisfies

$$
\partial_{t} U_{i}^{n}-A^{n} \Delta U_{i}^{n}=A^{n} v^{n}, \partial_{n} U_{i}^{n}=0 \text { on } \Sigma_{T}, U_{i}^{n}(0)=0 .
$$

By Lemma 4.2, there exists $\alpha, C$ independent of $n$ such that $\left\|U_{i}^{n}\right\|_{T}^{(\alpha)} \leq C$. By Lemma 2.3, $u_{i}^{n}$ converges to $u_{i}$ in $L^{2}\left(Q_{T}\right)$ which implies that $U_{i}^{n}$ also converges to $U_{i}$ in $L^{2}\left(Q_{T}\right)$. Whence the estimate of Proposition 4.4 on $U_{i}$. The estimate on $\tilde{u}_{i}=w_{i}-U_{i}$ follows by combining with Proposition 4.1 which says that $w_{i}$ is even Lipschitz continuous.

Now that we know that the coefficient $a_{i}(\tilde{u})$ is not only bounded but also continuous, we may continue improving the regularity of $u$.

Proposition 4.5 Assume $u^{0} \in L^{\infty}(\Omega,[0, \infty))^{I}$. Then,

$$
\forall p \in[1, \infty), \forall T>0, \forall i=1, \ldots, I, u_{i}, \partial_{t} U_{i}, \Delta U_{i} \in L^{p}\left(Q_{T}\right)
$$


Proof: We may formally write

$$
\partial_{t} U_{i}-a_{i}(\tilde{u}) \Delta U_{i}=a_{i}(\tilde{u}) u_{i}^{0}, \partial_{n} U_{i}=0, U_{i}(0)=0 .
$$

Here $a_{i}(\tilde{u})$ is continuous on $\overline{Q_{T}}$ so that, $a_{i}(\tilde{u})$ being given, this equation has a unique solution : let us call it $V_{i}$. We set $v_{i}:=\partial_{t} V_{i} / a_{i}(\tilde{u})$. Then

$$
v_{i}-\Delta V_{i}=u_{i}^{0}, V_{i}=\int_{0}^{t} a_{i}(\tilde{u}) v_{i}, \partial_{n}\left(\int_{0}^{t} a_{i}(\tilde{u}) v_{i}\right)=0 .
$$

Thus, $v_{i}$ coincides with our $u_{i}$ (and $V_{i}$ coincides with our $U_{i}$ ) thanks to the uniqueness result of Lemma 2.3 .

Moreover, $L^{p}$-maximal regularity holds for the equation $(50)$ since $a_{i}(\tilde{u})$ is continuous (see for instance [14], Theorem 9.1 or [21], Theorem 2.5.2) so that, as $a_{i}(\tilde{u}) u_{i}^{0} \in L^{\infty}\left(Q_{T}\right) \subset L^{p}\left(Q_{T}\right)$, we have

$$
\forall p \in(1, \infty),\left\|\partial_{t} U_{i}\right\|_{L^{p}\left(Q_{T}\right)},\left\|\Delta U_{i}\right\|_{L^{p}\left(Q_{T}\right)} \leq C,
$$

where $C$ depends on $p,\left\|a_{i}(\tilde{u}) u_{i}^{0}\right\|_{L^{\infty}\left(Q_{T}\right)}$ and on the modulus of continuity of the function $a_{i}(\tilde{u})$.

Next, from $0 \leq u_{i} \leq \underline{d}^{-1} a_{i}(\tilde{u}) u_{i}=\underline{d}^{-1}\left|\partial_{t} U_{i}\right|$, we deduce that $u_{i} \in L^{p}\left(Q_{T}\right)$ as well. And $p=1$ is also included since $Q_{T}$ is bounded.

With Proposition 4.5, the first part of the existence result in Theorem 1.1 is now complete. We will now assume that $a_{i}$ is locally Lipschitz continuous.

Proposition 4.6 Besides (3), assume $a_{i}$ is locally Lipschitz continuous for all $i=1, \ldots, I$. Assume also $u^{0} \in L^{\infty}(\Omega,[0, \infty))^{I}$. Then

$$
\forall i=1, \ldots, I, \forall T>0, u_{i} \in L^{\infty}\left(Q_{T}\right), \forall p<\infty, \forall \tau \in(0, T), \partial_{t} u_{i}, \Delta\left(a_{i}(\tilde{u}) u_{i}\right) \in L^{p}((\tau, T) \times \Omega),
$$

and

$$
\partial_{t} u_{i}-\Delta\left(a_{i}(\tilde{u}) u_{i}\right)=0 \text { on } Q_{T}, \partial_{n}\left(a_{i}(\tilde{u}) u_{i}\right)=0 \text { on } \Sigma_{T}
$$

is satisfied pointwise.

Proof: The equation in $u_{i}$ may also be written (at least formally to start):

$$
\partial_{t}\left(a_{i}(\tilde{u}) u_{i}\right)-a_{i}(\tilde{u}) \Delta\left(a_{i}(\tilde{u}) u_{i}\right)=u_{i} D a_{i}(\tilde{u}) \cdot \partial_{t} \tilde{u} .
$$

We know that $\delta_{i} \partial_{t} \tilde{u}_{i}+a_{i}(\tilde{u}) u_{i} \in L^{\infty}\left(Q_{T}\right)$ (see Proposition 4.1), and $a_{i}(\tilde{u}) u_{i} \in L^{p}\left(Q_{T}\right)$, for all $p<\infty$, so that $\partial_{t} \tilde{u}_{i} \in L^{p}\left(Q_{T}\right)$ for all $p<\infty$. The right hand side of this equation $F:=u_{i} D a_{i}(\tilde{u}) \cdot \partial_{t} \tilde{u}$ is therefore in $L^{p}\left(Q_{T}\right)$ for all $p<\infty$ since also $D a_{i}(\tilde{u}) \in L^{\infty}\left(Q_{T}\right)^{N}$ (as $a_{i}$ is locally Lipschitz and $\tilde{u}$ is bounded).

Since $a_{i}(\tilde{u})$ is continuous on $\overline{Q_{T}}$, we know (see again e.g. [14], Theorem 9.1 or [21], Theorem 2.5.2), there exists a (unique) solution $\theta$ to

$$
\left\{\begin{array}{l}
\forall p<\infty, \theta \in C\left(0, T ; L^{p}(\Omega)\right), \forall \tau \in(0, T), \partial_{t} \theta, \Delta \theta \in L^{p}((\tau, T) \times \Omega) \\
\partial_{t} \theta-a_{i}(\tilde{u}) \Delta \theta=F, \partial_{n} \theta=0 \text { on } \Sigma_{T}, \theta(0)=a_{i}\left(\tilde{u}^{0}\right) u_{i}^{0}
\end{array}\right.
$$

and we have

$$
\|\theta\|_{L^{\infty}\left(Q_{T}\right)}+\left\|\partial_{t} \theta\right\|_{L^{p}((\tau, T) \times \Omega)}+\|\Delta \theta\|_{L^{p}((\tau, T) \times \Omega)} \leq C\left[\|F\|_{L^{p}\left(Q_{T}\right)}+\left\|u_{i}^{0}\right\|_{L^{\infty}(\Omega)}\right]
$$


where $C$ depends on $\tau, T, p, \Omega$ and of the modulus of continuity of $a_{i}(\tilde{u})$.

If we knew that $\theta=a_{i}(\tilde{u}) u_{i}$, then the proof of Proposition 4.6 would be complete using moreover:

$$
\partial_{t} u_{i}=a_{i}(\tilde{u})^{-1}\left[\partial_{t}\left(a_{i}(\tilde{u}) u_{i}\right)-u_{i} D a_{i}(\tilde{u}) \cdot \partial_{t} \tilde{u}\right] \in L^{p}((\tau, T) \times \Omega)
$$

To prove it, we recall (see the proof of Lemma 2.3) that $u_{i}$ is the limit of the approximate solutions $u^{n}$ of

$$
\partial_{t} u^{n}-\Delta\left(A^{n} u^{n}\right)=0, \partial_{n} u^{n}=0 \text { on } \Sigma_{T}, u^{n}(0)=u_{i}^{0},
$$

where $A^{n}$ is smooth and converges pointwise to $a_{i}(\tilde{u})$ with $0<\min a_{i}(\tilde{u}) \leq A^{n} \leq \max a_{i}(\tilde{u})<+\infty$. Moreover, $u^{n}$ is bounded in $L^{p}\left(Q_{T}\right)^{I}$ for all $p<\infty$ by the analysis in Proposition 4.5. Here, we choose such an approximation $A^{n}$ which moreover satisfies

$$
A^{n} \rightarrow a_{i}(\tilde{u}) \text { in } L^{\infty}\left(Q_{T}\right), \partial_{t} A^{n} \rightarrow \partial_{t} a_{i}(\tilde{u})=D a_{i}(\tilde{u}) \cdot \partial_{t} \tilde{u} \text { in } L^{p}\left(Q_{T}\right) \forall p<\infty .
$$

Then, we apply the estimates (53) to $A^{n} u^{n}$ which satisfies

$$
\partial_{t}\left(A^{n} u^{n}\right)-A^{n} \Delta\left(A^{n} u^{n}\right)=u^{n} \partial_{t} A^{n}, \partial_{n} A^{n}=0 \text { on } \Sigma_{T}, A^{n} u^{n}(0)=a_{i}\left(\tilde{u}^{0}\right) u_{i}^{0},
$$

and they are preserved at the limit. Whence $\theta=a_{i}(\tilde{u}) u_{i}$ by uniqueness in (52).

Proof of the Existence in Theorem 1.1: It is a consequence of Corollary 3.5 and of Propositions $4.4,4.5,4.6$.

Remark 4.7 Note that, not only we proved existence of a solution with the announced regularity, but we even proved that any weak solution as in Corollary 3.5 has actually the announced regularity. This will be useful in the proof of uniqueness

Remark 4.8 The assumption that the $a_{i}$ are bounded from below is essential in our proof of existence, first for the $L^{2}$-estimate, next to apply the Krylov-Safonov regularity theory. In the case when the $a_{i}$ degenerate $\left(a_{i} \geq 0\right)$, the $L^{2}$ a priori estimate is to be replaced by $\sqrt{a_{i}(\tilde{u})} u_{i} \in L^{2}\left(Q_{T}\right)$. However, we loose the $L^{2}$-compactness of the approximate solutions and also most regularity properties of the solution as well. It could however be interesting to study the possibility of existence of weak solutions.

Remark 4.9 The above analysis relies on the use of the $C^{\alpha}$-Krylov-Safonov estimates. However, it is interesting to notice that one can prove directly, by an elementary estimate, that $u \in L^{\infty}\left(Q_{T}\right)$, without using these estimates in the (rather general situation) where, besides (3), $a_{i}$ satisfies

$$
\forall i=1, \ldots, I, a_{i} \text { is locally Lipschitz continuous, } \forall j=1, \ldots, I, \partial_{\tilde{u}_{j}} a_{i} \geq 0 .
$$

Once the $L^{\infty}$-estimate is proved on $u_{i}$, the full regularity follows by the same arguments as in Proposition 4.6.

We indicate below (at least formally) the computations which leads to $u \in L^{\infty}\left(Q_{T}\right)$.

Proof of $u \in L^{\infty}\left(Q_{T}\right)$ under assumption (54):

We write $a_{i}$ for $a_{i}(\tilde{u})$ and $a_{i j}=\partial_{\tilde{u}_{j}} a_{i}$. We multiply the equation $\partial_{t} u_{i}-\Delta\left(a_{i} u_{i}\right)=0$ by $p\left(a_{i} u_{i}\right)^{p-1}$ and we integrate over $\Omega$ :

$$
\frac{d}{d t} \int_{\Omega} a_{i}^{p-1} u_{i}^{p}+\int_{\Omega} p(p-1)\left(a_{i} u_{i}\right)^{p-2}\left|\nabla\left(a_{i} u_{i}\right)\right|^{2}=(p-1) \sum_{j} \int_{\Omega} a_{i}^{p-2} u_{i}^{p} a_{i j} \partial_{t} \tilde{u}_{j}
$$


We proved in Proposition 4.1 that $\partial_{t} \tilde{u}_{j}+a_{j} u_{j} \leq C(T)<\infty$. This implies $\partial_{t} \tilde{u}_{j} \leq C(T)$. Plugging this into (55), using $a_{i j} \geq 0, a_{i j}$ bounded and $a_{i} \geq \underline{d}$ leads with some $C_{T}$ independent of $p$ to:

$$
\frac{d}{d t} \int_{\Omega} a_{i}^{p-1} u_{i}^{p}+\int_{\Omega} p(p-1)\left(a_{i} u_{i}\right)^{p-2}\left|\nabla\left(a_{i} u_{i}\right)\right|^{2} \leq C_{T}(p-1) \sum_{j} \int_{\Omega} a_{i}^{p-1} u_{i}^{p} .
$$

Summing over $i$ and using Gronwall's lemma on the term $\sum_{i} \int_{\Omega} a_{i}^{p-1} u_{i}^{p}$, we then have

$$
\sum_{i} \int_{\Omega} a_{i}^{p-1} u_{i}^{p}(t) \leq e^{I T C_{T}(p-1)} \sum_{i} \int_{\Omega} a_{i}^{p-1} u_{i}^{p}(0) .
$$

Using the lower and upper bounds on $a_{i}$, we have with $A, C_{T}^{1}$ both independent of $p$ :

$$
\sum_{i} \int_{\Omega} a_{i}^{p} u_{i}^{p}(t) \leq A e^{C_{T}^{1} p}\left(1+\sum_{i}\left\|a_{i} u_{i}(0)\right\|_{\infty}\right)^{p}
$$

This implies

$$
\left\|\left(a_{i} u_{i}\right)(t)\right\|_{p} \leq A^{1 / p} e^{C_{T}^{1}}\left(1+\sum_{i}\left\|a_{i} u_{i}(0)\right\|_{\infty}\right)
$$

whence the $L^{\infty}$-estimate on $a_{i} u_{i}$ by letting $p \rightarrow \infty$, and then on $u_{i}$ itself by using the lower bound on $a_{i}$.

\section{Proof of uniqueness in Theorem 1.1}

Actually, we will prove the more general following result:

Proposition 5.1 Let $u^{0} \in L^{\infty}(\Omega,[0, \infty))^{I}$. Assume that for all $i=1, \ldots, I, a_{i}$ satisfies (3) and is locally Lipschitz continuous. Then there exists a unique solution to the system (42) in Corollary 3.5.

Proof: By Remark 4.7, we already know that any solution of (42) satisfies the regularity stated in Proposition 4.6 and Theorem 1.1. Let $u, v$ be two such solutions. We denote $a_{i}=a_{i}(\tilde{u}), b_{i}=a_{i}(\tilde{v})$. By difference,

$$
\partial_{t}\left(u_{i}-v_{i}\right)-\Delta\left[a_{i}\left(u_{i}-v_{i}\right)+v_{i}\left(a_{i}-b_{i}\right)\right]=0 .
$$

We set

$$
U_{i}=u_{i}-v_{i}, \tilde{U}_{i}=\tilde{u}_{i}-\tilde{v}_{i}, \widetilde{U}=\tilde{u}-\tilde{v}, A_{i}=\int_{0}^{1} D a_{i}(t \tilde{u}+(1-t) \tilde{v}) d t
$$

so that $a_{i}-b_{i}=A_{i} \cdot(\tilde{u}-\tilde{v})=\sum_{j} A_{i j} \tilde{U}_{j}$. Note that $\left\|A_{i}\right\|_{L^{\infty}}<\infty$. Then

$$
\partial_{t} U_{i}-\Delta\left[a_{i} U_{i}+v_{i} A_{i} \cdot \widetilde{U}\right]=0, \partial_{\nu}\left(a_{i} U_{i}+v_{i} A_{i} \cdot \widetilde{U}\right)=0 .
$$

Lemma 5.2 Let $F \in C_{0}^{\infty}\left(Q_{T}\right)^{I}$. There exists a solution to the dual problem

$$
\left\{\begin{array}{l}
\forall i=1, \ldots, I, \varphi_{i}, \partial_{t} \varphi_{i}, \Delta \varphi_{i} \in L^{2}\left(Q_{T}\right) \\
\partial_{t} \varphi_{i}+a_{i} \Delta \varphi_{i}+J_{\delta_{i}}\left(B_{i} \cdot \Delta \varphi\right)=F_{i} \text { on } Q_{T} \\
\varphi=\left(\varphi_{1}, \ldots, \varphi_{I}\right), \partial_{\nu} \varphi_{i}=0 \text { on } \Sigma_{T}, \varphi_{i}(T)=0
\end{array}\right.
$$

where $B_{i}=\left(B_{i 1}, \ldots, B_{i I}\right), B_{i j}=v_{j} A_{j i}$. 
Assuming this lemma, we multiply each equation (56) by $\varphi_{i}$ and we obtain after integration on $Q_{T}$ (the integrations by parts are allowed, thanks to the regularity of $u, v, \tilde{u}, \tilde{v}, \varphi_{i}$ and the boundary conditions; we also use $\left.\int_{Q_{T}} U_{i} J_{\delta_{i}}\left(B_{i} \cdot \Delta \varphi\right)=\int_{Q_{T}} \widetilde{U}_{i} B_{i} \cdot \Delta \varphi\right)$ :

$$
0=\int_{Q_{T}} U_{i}\left[\partial_{t} \varphi_{i}+a_{i} \Delta \varphi_{i}\right]+\Delta \varphi_{i} v_{i} A_{i} \cdot \tilde{U}=\int_{Q_{T}} U_{i} F_{i}-\tilde{U}_{i} B_{i} \cdot \Delta \varphi+\Delta \varphi_{i} v_{i} A_{i} \cdot \tilde{U}
$$

Summing these $I$ identities gives $\sum_{i} \int_{Q_{T}} U_{i} F_{i}=0$ which implies $U \equiv 0$ by arbitrarity of the $F_{i}$, whence uniqueness.

Proof of Lemma 5.2: To solve the dual problem (actually interesting for itself), we may start with $a_{i}$ replaced by regular approximations $A_{i}^{n}$ converging in the usual way to $a_{i}$ (which means a.e. and uniformly bounded from above and from below), and we first solve

$$
\partial_{t} \theta_{i}^{n}+\Delta\left(A_{i}^{n} \theta_{i}^{n}\right)+\Delta J_{\delta_{i}}\left(B_{i} \cdot \theta^{n}\right)=\Delta F_{i}, \partial_{n}\left(A_{i}^{n} \theta_{i}^{n}\right)=0, \theta_{i}(T)=0 .
$$

This is possible since $\theta \in L^{2}\left(Q_{T}\right)^{I} \rightarrow\left(\Delta J_{\delta_{i}}\left(B_{i} \cdot \theta\right)\right)_{1 \leq i \leq I} \in L^{2}\left(Q_{T}\right)^{I}$ is a Lipschitz perturbation (recall that $B_{i} \in L^{\infty}$ and $\Delta J_{\delta_{i}}$ is the Yosida approximation of the operator $-\Delta$ with homogeneous Neumann boundary conditions). Note that $\int_{\Omega} \theta_{i}^{n}(t)=0$. Next, we solve

$$
\Delta \varphi_{i}^{n}=\theta_{i}^{n} \text { in } \Omega, \partial_{n}\left(\varphi_{i}^{n}\right)=0 \text { on } \partial \Omega, \int_{\Omega} \phi_{i}^{n}=0
$$

so that, "by applying $\Delta^{-1}$ " to the equation in $\theta_{i}^{n}$, we obtain

$$
\partial_{t} \varphi_{i}^{n}+A_{i}^{n} \Delta \varphi_{i}^{n}+J_{\delta_{i}}\left(B_{i} \cdot \Delta \varphi^{n}\right)=F_{i}, \partial_{n}\left(\varphi_{i}^{n}\right)=0 \text { on } \Sigma_{T}, \varphi_{i}^{n}(T)=0 .
$$

Next, multiplying by $\Delta \varphi_{i}^{n}$ gives

$$
\int_{\Omega}-\frac{1}{2} \partial_{t}\left|\nabla \varphi_{i}^{n}\right|^{2}+A_{i}^{n}\left(\Delta \varphi_{i}^{n}\right)^{2}+\Delta \varphi_{i}^{n} J_{\delta_{i}}\left(B_{i} \cdot \Delta \varphi^{n}\right)=\int_{\Omega} F_{i} \Delta \varphi_{i}^{n} \leq \int_{\Omega} \epsilon\left(\Delta \varphi_{i}^{n}\right)^{2}+C_{\epsilon} F_{i}^{2} .
$$

We choose $\epsilon:=\underline{d} / 2$ and we deduce

$$
\int_{\Omega}-\frac{1}{2} \partial_{t}\left|\nabla \varphi_{i}^{n}\right|^{2}+\frac{d}{2}\left(\Delta \varphi_{i}^{n}\right)^{2} \leq C \int_{\Omega} F_{i}^{2}+\int_{\Omega} \nabla Z \nabla \varphi_{i}^{n} \leq C \int_{\Omega} F_{i}^{2}+\int_{\Omega} \epsilon|\nabla Z|^{2}+C_{\epsilon}\left|\nabla \varphi_{i}^{n}\right|^{2},
$$

where $Z-\delta_{i} \Delta Z=B_{i} \cdot \Delta \varphi^{n}, \partial_{n} Z=0$. Multipling this by $Z$ gives

$$
\int_{\Omega} Z^{2}+\delta_{i}|\nabla Z|^{2}=\int_{\Omega} Z B_{i} \cdot \Delta \varphi^{n} \leq\left\|B_{i}\right\|_{L^{\infty}} \int_{\Omega} \epsilon Z^{2}+C_{\epsilon}\left|\Delta \varphi^{n}\right|^{2} \Rightarrow \int_{\Omega}|\nabla Z|^{2} \leq C \int_{\Omega}\left|\Delta \varphi^{n}\right|^{2} .
$$

Summing the equations in (59) and choosing adequately $\epsilon$ leads to (with a different $C$ )

$$
-\partial_{t} \int_{\Omega} \sum_{i}\left|\nabla \varphi_{i}^{n}\right|^{2}+\frac{d}{2} \sum_{i} \int_{\Omega}\left(\Delta \varphi_{i}^{n}\right)^{2} \leq C\left[\int_{\Omega} \sum_{i}\left[F_{i}^{2}+\left|\nabla \varphi_{i}^{n}\right|^{2}\right]\right] .
$$

Integrating the Gronwall estimate in $\sum_{i}\left|\nabla \varphi_{i}^{n}\right|^{2}$ and plugging back the terms in $\Delta \varphi_{i}^{n}$ yield

$$
\sup _{0 \leq t \leq T} \int_{\Omega} \sum_{i}\left|\nabla \varphi_{i}^{n}\right|^{2}+\frac{d}{2} \int_{Q_{T}}\left|\Delta \varphi^{n}\right|^{2} \leq C \int_{Q_{T}}|F|^{2} .
$$

By going back to (58), we also obtain that $\partial_{t} \varphi_{i}^{n}$ is bounded in $L^{2}\left(Q_{T}\right)$. Now, we can pass to the limit as $n \rightarrow \infty$, weakly in $L^{2}\left(Q_{T}\right)$ in each term of (58), to prove the existence result of Lemma 5.2. 
Remark 5.3 We do not know whether uniqueness holds or not without assuming Lipschitz continuity of the $a_{i}$. The above proof indicates that uniqueness is essentially equivalent to solving the "dual" problem (57). The fact that $B_{i} \in L^{\infty}\left(Q_{T}\right)$ (which equivalent to the Lipschitz continuity of $a_{i}$ ) is strongly used in the estimates to solve (57). It is not clear how to weaken it.

\section{A constructive approximation procedure}

In this Section, we just mention without proof a complementary approach for the proof of existence and regularity of solutions to our system. A interesting point is that it provides a constructive approach and may be used to provide a numerical approximation schemes of the solutions (and have actually been used in [15]).

The approximation procedure follows the ideas of [15] to make a time semi-discretization with an explicit treatment of $\tilde{u}$ and an implicit treatment of $u_{i}$ in $a_{i}(\tilde{u}) u_{i}$. We fix $T>0$ in the following. Let $\tau>0$ is given. We introduce the following approximate system

$$
\left\{\begin{array}{l}
\frac{u_{i}^{n+1}-u_{i}^{n}}{\tau}-\Delta a_{i}\left[\left(\tilde{u}^{n}\right) u_{i}^{n+1}\right]=0, \quad \text { in } \Omega, \\
-\delta_{i} \Delta \tilde{u}_{i}^{n}+\tilde{u}_{i}^{n}=u_{i}^{n}, \quad \text { in } \Omega, \\
\partial_{n} \tilde{u}_{i}^{n}=\partial_{n} u_{i}^{n+1}=0, \quad \text { on } \partial \Omega .
\end{array}\right.
$$

Then, using some adequate discretized version of the approaches in Section 3, it may be proved that: - there exists a unique solution to the system (60),

- the discrete version of the $L^{1}$-estimate (41) is valid for $B^{n}=\sum_{i} a_{i}\left(\tilde{u}^{n}\right) u_{i}^{n+1}$,

- an $L^{\infty}$-estimate may be deduced for $\tilde{u}^{n}$,

- the direct $L^{\infty}$ estimate may be reproduced in this direct situation under the assumption (54). Once all these estimates are obtained at the discrete level, one can get Corollary 3.5.

A common issue to both procedures is the need, first to truncate the coefficients (for steps 2 and 3), and then notice a posteriori that this truncation was useless.

Remark 6.1 We have used here a situation where the $i$-th relaxation parameter depends on the species (in words $\delta_{i}$ depends on $i$ ). It is quite natural on a modeling point of view to consider:

- situations where the relaxation parameter might depend on the species that are observed and on the species that are observing (in words, $\left.a_{i}=a_{i}\left(J_{\delta_{i 1}}\left(u_{1}\right), \ldots, J_{\delta_{i I}}\left(u_{I}\right)\right)\right)$.

- several sensitivities for a single species might have to be considered, short and long distances could matter in the diffusion coefficient (in words two or more different $J_{\delta}\left(u_{j}\right)$ could be taken into consideration in $a_{i}$ ).

Our results could be easily extended to these more general situations through rather slight modifications.

\section{References}

[1] B.Andreinnov, M. Bendahmane, And R. R. Baier, Analysis of a finite volume method for a crossdiffusion model in population dynamics, M3AS Math. Models Methods Appl. Sci., 21 (2011), pp. 307-344. 
[2] M. Bendahmane And M. Langlais, A one-dimensional model of cell diffusion and aggregation, incorporating volume filling and cell-to-cell adhesion, J. of Evol. Equ., 10 (2010), pp. 883-904.

[3] M. Bendahmane, T. Lepoutre, A. Marrocco, and B. Perthame, Conservative cross diffusions and pattern formation through relaxation, Journal de Mathématiques Pures et Appliquées, 92 (2009), pp. $651-66 \%$.

[4] M. Bendahmane and M. Sepulveda, Convergence of a finite volume scheme for nonlocal reaction-diffusion systems modelling an epidemic disease, Discrete and Cont. Dyn. Systems, Series $B, 11$ (2009), pp. 823-853.

[5] H. Berestycki, G. Nadin, B. Perthame, and L. Ryzhik, The non-local Fisher-KPP equation: travelling waves and steady states, Nonlinearity, 22 (2009), p. 2813.

[6] H. Brezis, Functional analysis, Sobolev Spaces and Partial Differential Equations, Universitext, Springer, New York, 2011.

[7] L. Chen And A. Jüngel, Analysis of a parabolic cross-diffusion population model without self-diffusion, J. Differential Equations, 224 (2006), pp. 39-59.

[8] S. Genieys, V. Volpert, and P. Auger, Pattern and waves for a model in population dynamics with nonlocal consumption of resources, Mathematical Modelling of Natural Phenomena, 1 (2006), pp. 63-80.

[9] D. Gilbarg and N. S. Trudinger, Elliptic partial differential equations of second order, Springer-Verlag, Berlin, 1977. Grundlehren der Mathematischen Wissenschaften, Vol. 224.

[10] D. Guangchang, Initial and nonlinear oblique boundary value problem for fully nonlinear parabolic equations, Journal of Partial Differential Equations, 1 (1988), pp. 12-42.

[11] M. Iida, M. Mimura, And H. Ninomiya, Diffusion, cross-diffusion and competitive interaction, J. Math. Biol., 53 (2006), pp. 617-641.

[12] A. Jüngel, Diffusive and nondiffusive population models, in: G. Naldi, L. Paresch and G. Toscani (eds.), Mathematical Modeling of Collective Behavior in Socio-Economic and Life Sciences, (2010).

[13] N. KRYLOv, Nonlinear elliptic and parabolic equations of the second order, Mathematics and its applications (D. Reidel Publishing Company): Soviet series, D. Reidel Pub. Co., $198 \%$.

[14] G. Ladyzhenskaya, V. Solonnikov, and N. Uralceva, Linear and Quasilinear Equations of Parabolic Type, Translations of Mathematical Monographs, American Mathematical Society, Providence, Rhode Island, 1968.

[15] T. Lepoutre, Analyse et modélisation de phénomènes de croissance et mouvement issus de la biologie, PhD thesis, Université Pierre et Marie Curie, November 2009.

[16] T. Lepoutre and S. Martinez, Steady states of relaxed cross diffusion models. In preparation.

[17] S. Levin and L. Segel, Hypothesis for origin of planktonic patchiness, Nature, 259 (1976), p. 659. 
[18] S. A. Levin, A more functional response to predator-prey stability, The American Naturalist, 108 (1977), pp. 207-228.

[19] Y. Li AND C. ZhaO, Global existence of solutions to a cross-diffusion system in higher dimensional domains, Discrete Contin. Dyn. Syst., 12 (2005), pp. 185-192.

[20] G. Lieberman, Second order parabolic differential equations, World Scientific, 2005.

[21] A. Maugeri, D. Palagachev, and L. Softova, Elliptic and Parabolic Equations with discontinuous coefficients, vol. 109, Wiley-Vch, Math. Research, 2000.

[22] M. Mimura And K. KaWASAKi, Spatial segregation in competitive interaction-diffusion equations, J. Math. Biol., 9 (1980), pp. 49-64.

[23] M. Mimura and J. D. Murray, On a diffusive prey-predator model which exhibits patchiness, J. Theoret. Biol., 75 (1978), pp. 249-262.

[24] M. Mimura and M. Yamaguti, Pattern formation in interacting and diffusing systems in population biology., Adv Biophys, 15 (1982), pp. 19-65.

[25] G. Nadin, B. Perthame, and M. Tang, Can a traveling wave connect two unstable states? The case of the nonlocal Fisher equation, Comptes Rendus Mathématiques, 349 (2011), pp. 553 $-55 \%$.

[26] A. Okubo, Diffusion and ecological problems: mathematical models, vol. 10 of Biomathematics, Springer-Verlag, Berlin, 1980. An extended version of the Japanese edition, it Ecology and diffusion, Translated by G. N. Parker.

[27] M. Pierre, Global existence in reaction-diffusion systems with control of mass: a Survey, Milan Journal of Mathematics, 78 (2010), pp. 417-455.

[28] N. Shigesada, K. Kawasaki, and E. Teramoto, Spatial segregation of interacting species, J. Theoret. Biol., 79 (1979), pp. 83-99.

[29] Y. Wang, The global existence of solutions for a cross-diffusion system, Acta Math. Appl. Sin. Engl. Ser., 21 (2005), pp. 519-528.

Thomas Lepoutre

INRIA Grenoble Rhône-Alpes, team DRACULA

Institut Camille Jordan UMR 5208

Université Claude Bernard Lyon 1

43 boulevard du 11 novembre 1918

69622 Villeurbanne cedex (France)

thomas.lepoutre@inria.fr

Michel Pierr

ENS Cachan Bretagne, IRMAR, EUB

Campus de Ker Lann

35170 Bruz (France)

michel.pierre@bretagne.ens-cachan.fr

Guillaume Rolland

ENS Cachan Bretagne, IRMAR, EUB

Campus de Ker Lann

35170 Bruz (France)

guillaume.rolland@bretagne.ens-cachan.fr 